\title{
Cervical Lymphadenopathy
}

National Cancer Institute

\section{Source}

National Cancer Institute. Cervical Lymphadenopathy. NCI Thesaurus. Code C40989.

Enlargement of the cervical lymph nodes usually due to infections or involvement by malignant neoplasms. 